Polibotánica

ISSN electrónico: 2395-9525

POLIBETÁNICA polibotanica@gmail.com Instituto Politécnico Nacional México http:www.polibotanica.mx

\title{
DIAGNÓSTICO DE LOS USOS, LA DISTRIBUCIÓN Y CARACTERÍSTICAS DASOMÉTRICAS DEL ÁRBOL CIRIÁN (Crescentia alata Kunth) EN EL MUNICIPIO DE PUNGARABATO, GUERRERO, MÉXICO
}

\section{DIAGNOSTIC OF USES, DISTRIBUTION AND DASOMETRIC CHARACTERISTICS OF THE CIRIAN (Crescentia alata Kunth) TREE IN PUNGARABATO MUNICIPALITY, GUERRERO, MEXICO}

Olivares-Pérez, J.; S. Rojas-Hernández, F. Quiroz-Cardozo, L.M. Camacho-Díaz, M. Cipriano-Salazar, M.A. Damián-Valdez, B. Ávila-Morales, y A. Villa-Mancera DIAGNÓSTICO DE LOS USOS, LA DISTRIBUCIÓN Y CARACTERÍSTICAS DASOMÉTRICAS DEL ÁRBOL CIRIÁN (Crescentia alata Kunth) EN EL MUNICIPIO DE PUNGARABATO, GUERRERO, MÉXICO

DIAGNOSTIC OF USES, DISTRIBUTION AND DASOMETRIC CHARACTERISTICS OF THE CIRIAN (Crescentia alata Kunth) TREE IN PUNGARABATO MUNICIPALITY, GUERRERO, MEXICO

POLIBETÁNICA

Instituto Politécnico Nacional
Núm. 45: 191-204, México. Enero 2018

DOI: $10.18387 /$ polibotanica.45.14 
DIAGNÓSTICO DE LOS USOS, LA DISTRIBUCIÓN Y CARACTERÍSTICAS DASOMÉTRICAS DEL ÁRBOL CIRIÁN (Crescentia alata Kunth) EN EL MUNICIPIO DE PUNGARABATO, GUERRERO, MÉXICO

\section{DIAGNOSTIC OF USES, DISTRIBUTION AND DASOMETRIC CHARACTERISTICS OF THE CIRIAN (Crescentia alata Kunth) TREE IN PUNGARABATO MUNICIPALITY, GUERRERO, MEXICO}

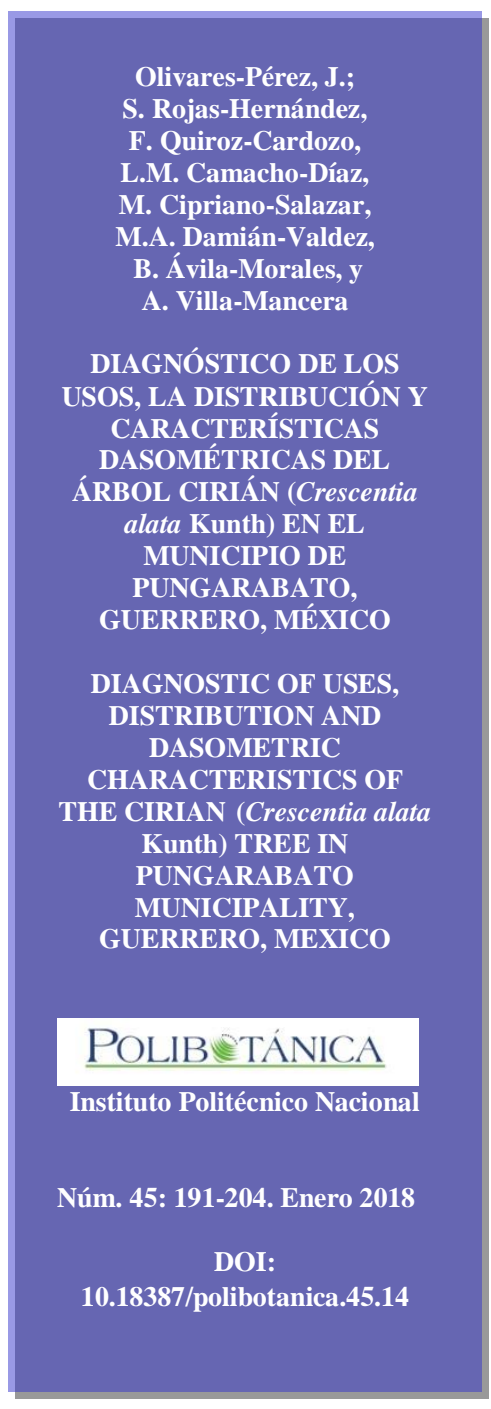

Olivares-Pérez, J.;

Rojas-Hernández,

Avila-Morales, $y$

DIAGNÓSTICO DE LOS

A DISTRIBUCION Y

CARACTERÍSTICAS

CAROMETRICAS DEL

alata Kunth) EN EL

MUNICIPIO DE

PUNGARABATO,

DIAGNOSTIC OF USES,

DISTRIBUTION AND

DASOMETRIC

CHARACTERISTICS OF

Kunth) TREE IN

PUNGARABATO

MUNICIPALITY,

GUERRERO, MEXICO

\section{POLIBETÁNICA}

Instituto Politécnico Nacional

Núm. 45: 191-204. Enero 2018

DOI:

10.18387/polibotanica.45.14

\author{
J. Olivares-Pérez \\ S. Rojas-Hernández/saulrh@ hotmail.com \\ F. Quiroz-Cardozo \\ L.M. Camacho-Díaz \\ M. Cipriano-Salazar \\ M.A. Damián-Valdéz \\ B. Ávila-Morales \\ Facultad de Medicina Veterinaria y Zootecnia núm.1 \\ Universidad Autónoma de Guerrero, México. \\ A. Villa-Mancera \\ Facultad de Veterinaria Benemérita \\ Universidad Autónoma de Puebla, México.
}

RESUMEN: El objetivo fue conocer el uso y aprovechamiento del árbol de Crescentia alata a través del conocimiento tradicional de los productores y amas de casa en el municipio de Pungarabato, Guerrero México. Se realizaron 140 encuestas a 70 ganaderos y 70 amas de casa. El 100\% de los productores conoce el árbol de Cirián y el $91.4 \%$ tienen este árbol en sus unidades de producción (UP), que se caracteriza por ser resistente al fuego $(52.9 \%)$ y al pisoteo $(81.4 \%)$, e inicia la producción de frutos entre los cinco y seis años $(44.3 \%)$. El mayor beneficio del árbol en la UP es la sombra (61.4\%), pero también aporta follaje y frutos para la alimentación del ganado (37.1\%). La madera es dura $(74.3 \%)$ y con la cáscara del fruto se elaboran jícaras (10\%), el uso ornamental es limitado (23.4\%). El fruto (100\%) se utiliza para tratar ocho enfermedades, sobresaliendo tos (52.9\%) y golpes (51.4\%). Con las hojas se elaboran tamales nejos $(48.6 \%)$ y con la semilla chocolate (7.1\%). El número de árboles dispersos en las praderas y en las cercas vivas fue de 218 y 224, respectivamente. La correlación fue positiva entre el peso $(0.657 \mathrm{~g})$ y el diámetro $(0.667 \mathrm{~cm})$ del fruto con el número de semillas $(\mathrm{P}<0.0001)$. C. alata es un árbol que aporta múltiples beneficios en las UP, sobresaliendo su uso en la herbolaria y en la alimentación de humanos y animales, por lo que es necesario promover su conservación y siembra.

Palabras claves: usos, herbolaria, beneficios, alimentación.

ABSTRACT: The objective was to know the use and utilization of Crescentia alata tree through the traditional knowledge of producers and housewives in the Municipality of Pungarabato, Guerrero Mexico. 140 surveys were carried out to 70 ranchers and 70 housewives. 100\% of the producers know the cirian tree and $91.4 \%$ have this tree in their production units (PU), which is characterized by being resistant to fire (52.9\%) and trampling (81.4\%) and start fruit production between five and six years $(44.3 \%)$. 
The greatest benefit in the UP of the tree is the shadow $(61.4 \%)$, but also brings foliage and fruit for livestock feed $(37.1 \%)$. The wood is hard $(74.3 \%)$ and the fruits shell is used for jícaras fabrication $(10 \%)$, the ornamental use is limited $(23.4 \%)$. The fruit $(100 \%)$ is used to treat eight diseases, standing out cough $(52.9 \%)$ and strokes $(51.4 \%)$. They are elaborated with the leaves tamales nejos $(48.6 \%)$ and chocolate with the seeds $(7.1 \%)$. The number dispersed trees in the prairies and in the live fences was 218 and 224, respectively. Positive correlation was observed between the weight $(0.657 \mathrm{~g})$ and the diameter $(0.667 \mathrm{~cm})$ of the fruit with the seeds number $(\mathrm{P}$ $<0.0001)$. C. alata is a tree that brings benefits multiple in the PU, being outstanding its use in the herbolaria and in the feeding of humans and animals, reason why it is necessary to promote its conservation and sowing.

Key words: uses, herbal, benefits, feed.

\section{INTRODUCCIÓN}

En México, la región tropical se caracteriza por su diversidad forrajera y representa el área con mayor potencial para el desarrollo de la ganadería, por ello la importancia de implementar sistemas de producción eficientes y sostenibles que estén en armonía con el medio ambiente (Palma, 2006). En la actualidad es importante revalorizar el conocimiento tradicional y los usos de las especies nativas, ya que es la base para su integración a los sistemas silvopastoriles. Este procedimiento ha sido desarrollado en varios sistemas donde se han reportado usos diversos como combustible (leña), madera, forraje, alimento para el ser humano (consumo de flores y frutos), medicinal (humanos), industrial (tintes y resinas), cercos (postes y cercas vivas) y sombra para el ganado (Harvey y Haber, 1999; Villacis et al., 2003; Couttolenc et al., 2005; Pinto et al., 2005; Olivares et al., 2016). En el estado de Guerrero existen árboles que podrían utilizarse en los sistemas de producción agropecuarios extensivos. Entre estos recursos está el árbol Crescentia alata Kunth (nombre común: cirián), que crece naturalmente en las sabanas del sur de México y América Central y que produce gran cantidad de frutos (Bass, 2004; Solares, 2004; Olivares et al., 2016), que pueden utilizarse en la medicina tradicional, alimentación de humanos y del ganado, por su aporte de energía, fibra, minerales y vitaminas (Hernández et al., 2002).

Figueroa y Bressani (2000), evaluaron la composición química del fruto del cirián indicando que el contenido de proteína cruda de la pulpa + semilla es de $18.6 \%$, en la pulpa de $12.9 \%$ y en la semilla de $26.4 \%$. La semilla contiene el mayor contenido de extracto etéreo (39.9\%) y la pulpa es la fracción que contiene mayor cantidad de cenizas (8.1\% en base seca). En otro estudio, Rojas et al. (2014) evaluaron la composición nutricional del ensilado de frutos (pulpa con semillas) de C. alata. El contenido de proteína fue de $15.5 \%$, de extracto etéreo el $13.8 \%$, la fibra detergente neutro de $53.2 \%$ y la fibra detergente ácido de $40.5 \%$, en tanto que el $\mathrm{pH}$ fue de 4.1. En el trópico seco de la región de Tierra Caliente de Guerrero existe gran diversidad de especies arbustivas distribuidas en pastizales, agostaderos y bosques, sin embargo, no han sido evaluados su contenido de nutrientes ni los usos en la alimentación humana, medicina tradicional, su potencial forrajero y la respuesta animal. Por lo anterior el objetivo del estudio fue conocer el uso y aprovechamiento del árbol de $C$. alata a través del conocimiento tradicional de los productores y amas de casa en el municipio de Pungarabato, Guerrero México.

\section{MATERIAL Y MÉTODOS}

El presente estudio se realizó en el municipio de Pungarabato, Guerrero, México, localizado en la región de la Tierra Caliente ( $18^{\circ} 20^{\prime} 30^{\prime}$ ' de latitud Norte y $100^{\circ} 39^{\prime} 18^{\prime}$ ' de longitud Oeste), a 250 m s.n.m. El clima predominante es el más seco de los cálidos subhúmedos (Aw $)$. La temperatura mínima y máxima anual de $28^{\circ} \mathrm{C}$ a $46^{\circ} \mathrm{C}$, con precipitación anual de $750 \mathrm{~mm}$ (Fragoso, 1990). 


\section{Uso y aprovechamiento del árbol Crescentia alata Kunth}

El municipio de Pungarabato cuenta con un censo de 269 productores agrupados en la Asociación Ganadera Local, de los que se seleccionaron 70 mediante la fórmula de Rojas (1987). La información sobre los diferentes usos de $C$. alata se obtuvo a partir de encuestas estructuradas aplicadas a los titulares de las Unidades de Producción (UP).

$$
\mathrm{n}=\frac{\frac{\mathrm{Z}^{2} \mathrm{q}}{\mathrm{E}^{2} \mathrm{p}}}{1+\frac{1}{\mathrm{~N}}\left[\frac{\mathrm{Z}^{2} \mathrm{q}}{\mathrm{E}^{2} \mathrm{p}}\right]-1}
$$

$\mathrm{Z}=$ nivel de confianza $95 \%$

$\mathrm{E}=$ nivel de precisión $10 \%$

$\mathrm{p}=$ variabilidad 0.5

$\mathrm{q}=$ variabilidad 0.5

(Rojas, 1987)

Con las encuestas se obtuvo la siguiente información: conoce el árbol de cirián, características por las que lo deja en la UP, meses del año en que los árboles producen flores y frutos, beneficios que obtiene del árbol y sus usos (alimentación animal, artesanal, maderable y ornamental).

Se aplicó una encuesta a 70 de mujeres (amas de casa) mayores de 40 años de edad, originarias y radicadas en la zona rural de estudio por ser las de mayor experiencia en los usos tradicionales de los árboles en la alimentación y en la herbolaria, para identificar los platillos que preparan y las enfermedades que tratan y/o controlan con las flores, frutos, hojas y corteza de C. alata.

\section{Número de árboles y medidas dasométricas}

El estudio se realizó en 600 ha pertenecientes a 14 unidades de producción. Las mediciones en campo se realizaron en 55 transectos de una hectárea para el diagnóstico de árboles dispersos en las praderas, y en 55 transectos de $100 \mathrm{~m}$ lineales trazados sobre cercos perimetrales y divisorios para contabilizar los árboles en arreglo de cercos vivos (Camacho, 2000). Asimismo, se registró el número de árboles adultos ( $>10 \mathrm{~cm}$ de DAP) dispersos en los potreros y en las cercas vivas se contabilizaron los árboles que sostuvieran o estuvieran en contacto con el alambre de púas (árboles $\mathrm{m}^{-100}$ lineales). De manera simultánea, en cada transecto se realizó la medición dasométrica de los árboles, la altura (h) se midió con un clinómetro electrónico marca Sunto, y el diámetro del fuste fue medido a $130 \mathrm{~cm}$ de altura de la base del suelo (DAP) (Camacho, 2000).

Para conocer la correlación entre el peso y diámetro del fruto con el número de semillas, se recolectaron 300 frutos maduros de 30 árboles, en el ejido de Los Limones perteneciente al municipio de Pungarabato.

\section{Análisis estadístico}

La información se concentró en una base de datos en el programa Excel y se analizaron mediante, estadística descriptiva (Steel y Torrie, 1988). La correlación se realizó en el programa estadístico SAS (2001) con un valor máximo de $\mathrm{P}=0.05$. 


\section{RESUlTADOS}

Conocimientos empíricos de ganaderos sobre el árbol Crescentia alata Kunth

La edad promedio de los productores fue de $55.6 \pm 15.3$ años, de los cuales en promedio han dedicado $33.4 \pm 16.6$ años de su vida a la actividad agropecuaria. El 100\% conoce el árbol y el 91.4\% tiene árboles en sus unidades de producción (UP), los cuales están distribuidos en terrenos agrícolas, dispersos en la pradera y en cercas vivas (gráfica 1).

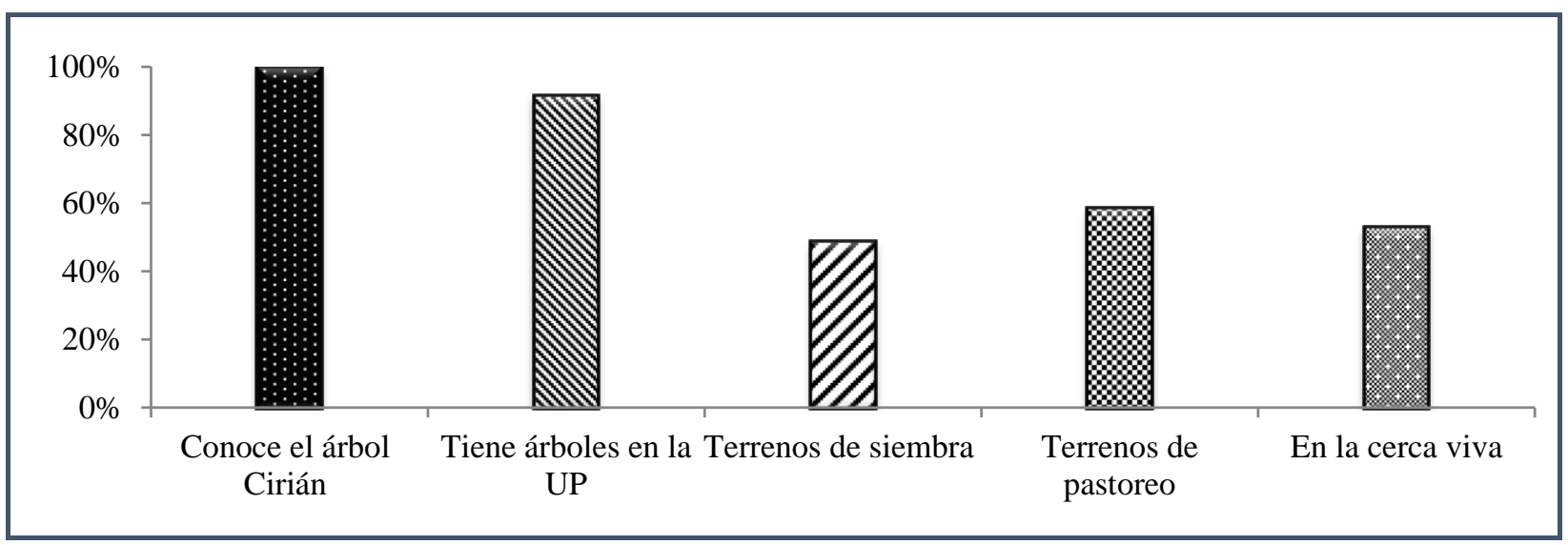

Gráfica 1. Conocimiento y distribución de C. alata en las unidades de producción (UP)

Meses del año en que hay producción de flores y fruto

Con base en sus conocimientos tradicionales los productores identificaron dos periodos de floración, el primero entre junio y julio (81.4\%) al inicio del periodo de lluvias, y durante septiembre y octubre (18.6\%) en las zonas de riego (gráfica 2), con dos periodos de producción de frutos de noviembre a enero (85.7\%) y de marzo a abril. El $44.3 \%$ de los productores coincide en que el árbol inicia su producción de frutos a una edad aproximada entre los cinco y seis años.

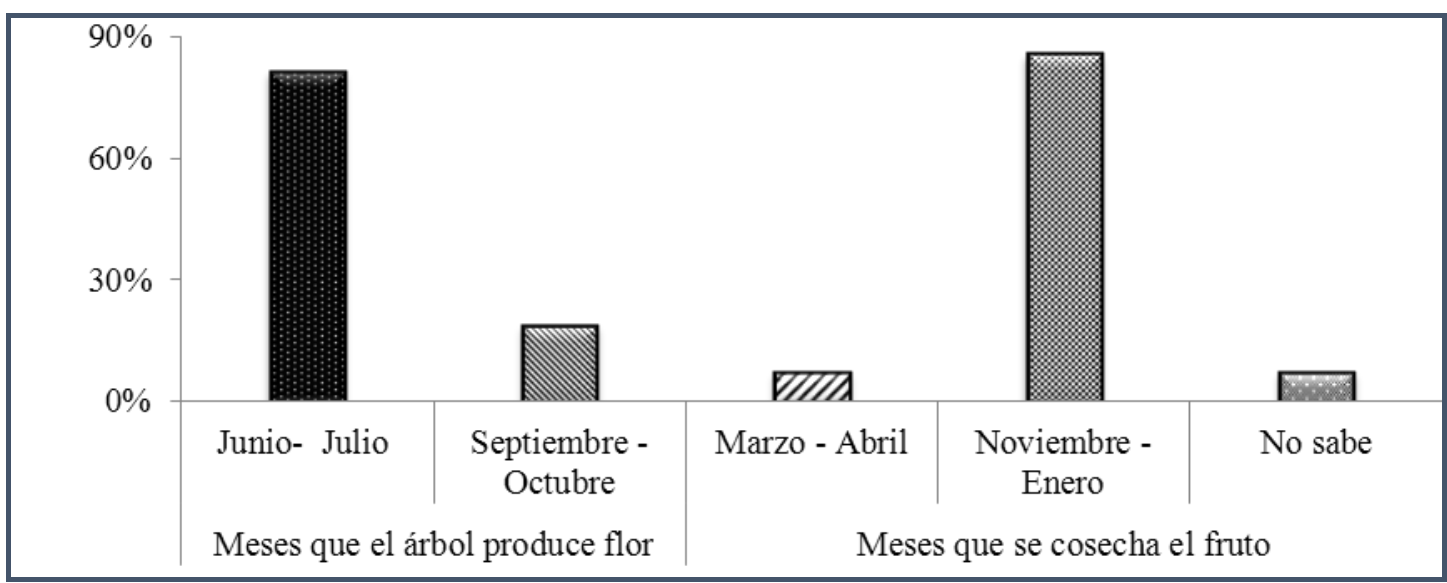

Gráfica 2. Conocimiento del periodo anual de floración y producción de fruto del árbol C. alata 


\section{Características, beneficios y uso en la alimentación animal}

Los árboles jóvenes de $C$. alata son resistente al pisoteo de los animales en las áreas de pastoreo (81.4\%), de igual manera los árboles jóvenes y adultos resisten al fuego (52.9\%), además proporciona sombra $(61.4 \%)$ y follaje (10.0\%) para el ganado, por estas características el $87.1 \%$ de los productores considera que es necesario conservar los árboles en las UP (cuadro 1).

Cuadro 1. Características, beneficios y uso en la alimentación animal del árbol C. alata.

\begin{tabular}{|c|c|c|}
\hline $\begin{array}{c}\text { Características por lo que es } \\
\text { conservado en las áreas de pastoreo }\end{array}$ & $\begin{array}{c}\text { Beneficios que } \\
\text { ofrece en las UP }\end{array}$ & Uso en la alimentación animal \\
\hline *Resisten el pisoteo $(81.4 \%)$ & Sombra $(61.4 \%)$ & Consumen el follaje y los frutos $(37.1 \%)$ \\
\hline **Resiste el fuego (52.9\%) & Fuente de follaje $(10 \%)$ & Corta follaje $(1.7 \%)$ \\
\hline ***Siembra árboles $(8.6 \%)$ & Medicinal (17.1\%) & Corta o almacena frutos $(0 \%)$ \\
\hline Conservación en los potreros $(87.1 \%)$ & Alimentación humana (1.4\%) & Elabora suplementos con follaje o frutos $(0 \%)$ \\
\hline Nace solo y resiste la sequía $(1.4 \%)$ & & Conoce que la pulpa contiene $16 \%$ de proteína \\
\hline \multirow[t]{2}{*}{ Está en peligro de extinción (5.7\%) } & & $(8.6 \%)$ \\
\hline & & $\begin{array}{l}\text { Fruto se conserva por medio de la técnica del } \\
\text { ensilaje }(18.6 \%)\end{array}$ \\
\hline
\end{tabular}

*Plántulas jóvenes de $\leq 50 \mathrm{~cm}$ de altura resisten el pisoteo del ganado. **Árboles con altura $\geq 1 \mathrm{~m}$ resisten la quema. ***Productores que siembran árboles de Crescentia alata en sus terrenos.

Uso artesanal, ornamental y maderable

El uso artesanal es mínimo (cuadro 2), destacando en orden de importancia la elaboración de jícaras (10.0\%) y sillas (7.1\%). El 23.3\% lo utiliza como árbol ornamental y el $18.6 \%$ consideran que los árboles lucen bonitos cuando están en la época de floración y adornan el paisaje.

Cuadro 2. Uso artesanal, ornamental y maderable de C. alata.

\begin{tabular}{lll}
\hline \multicolumn{1}{c}{ Artesanal } & \multicolumn{1}{c}{ Ornamental } & \multicolumn{1}{c}{ Maderable } \\
\hline *Jícaras $(10.0 \%)$ & Cuenta con árboles en su & La madera es dura (74.3\%) \\
Sillas $(7.1 \%)$ & domicilio $(23.3 \%)$ & Postes para cerca $(24.3 \%)$ \\
Comederos para animales $(4.2 \%)$ & Adornan el paisaje $(18.6 \%)$ & Mangos para herramientas $(21.4 \%)$ \\
Figuras $(2.8 \%)$ & & \\
Fustes para montar $(2.8 \%)$ & & \\
Tortilleros $(1.4 \%)$ & & \\
Mesas $(1.4 \%)$ & & \\
Trompos $(1.4 \%)$ & & \\
\hline
\end{tabular}

*Artesanía elaborada con el fruto de Crescentia alata pulido y decorado utilizado por la población como recipiente para tomar agua.

El productor considera que la madera es dura (74.3\%), pese a ello solo el $24.3 \%$ lo utiliza como poste en la cerca y el $18.6 \%$ considera que los postes tienen una vida media de uno a dos años y lo atribuyen a que no resiste la humedad del suelo. Debido a que es madera dura y ligera la población la utiliza para la elaboración de mangos para martillos, cabos para hachas (21.4\%), así como la fabricación de sillas y mesas $(8.6 \%)$ debido a que son utensilios que se aíslan del contacto con la humedad y en estas condiciones la vida útil puede ser superior a los cinco años. 


\section{Uso en la alimentación de humanos}

En la gastronomía regional las mujeres emplean las hojas y los frutos en la preparación de comidas, bebidas y dulces (gráfica 3). Las hojas y tallos tiernos son utilizadas en un $48.6 \%$ para dar sabor al tamal nejo (producto elaborado con masa obtenida dela molienda del grano de maíz previamente cocido en un medio acuoso con ceniza y cal hidratada, donde la masa es moldeada en hojas de plátano y se pone a cocer en un medio acuoso adicionado con tallos y hojas de $C$. alata durante un promedio de 60 minutos) que sustituye a la tortilla en un platillo denominado mole calentano típico de la región, con las semillas tostadas y molidas se le agrega agua caliente para preparar una bebida que en ámbito local se conoce como chocolate de cirián (7.1\%), con las semillas tostadas y molidas se prepara agua fresca $(2.9 \%)$, mientras que la pulpa del fruto se mezcla con azúcar, miel de abeja o piloncillo de caña, para elaborar un dulce denominado miel de cirián (2.9\%). Por lo anterior, es importante conservar el árbol de C. alata por el uso tradicional en la alimentación que en muchas ocasiones representa fuente de alimento y/o ingresos económicos para las familias por la venta de subproductos artesanales como jícaras, sillas, fustes para montura, tortilleros, trompos y gastronómicos como el tamal nejo, miel de cirián, chocolate de cirián.

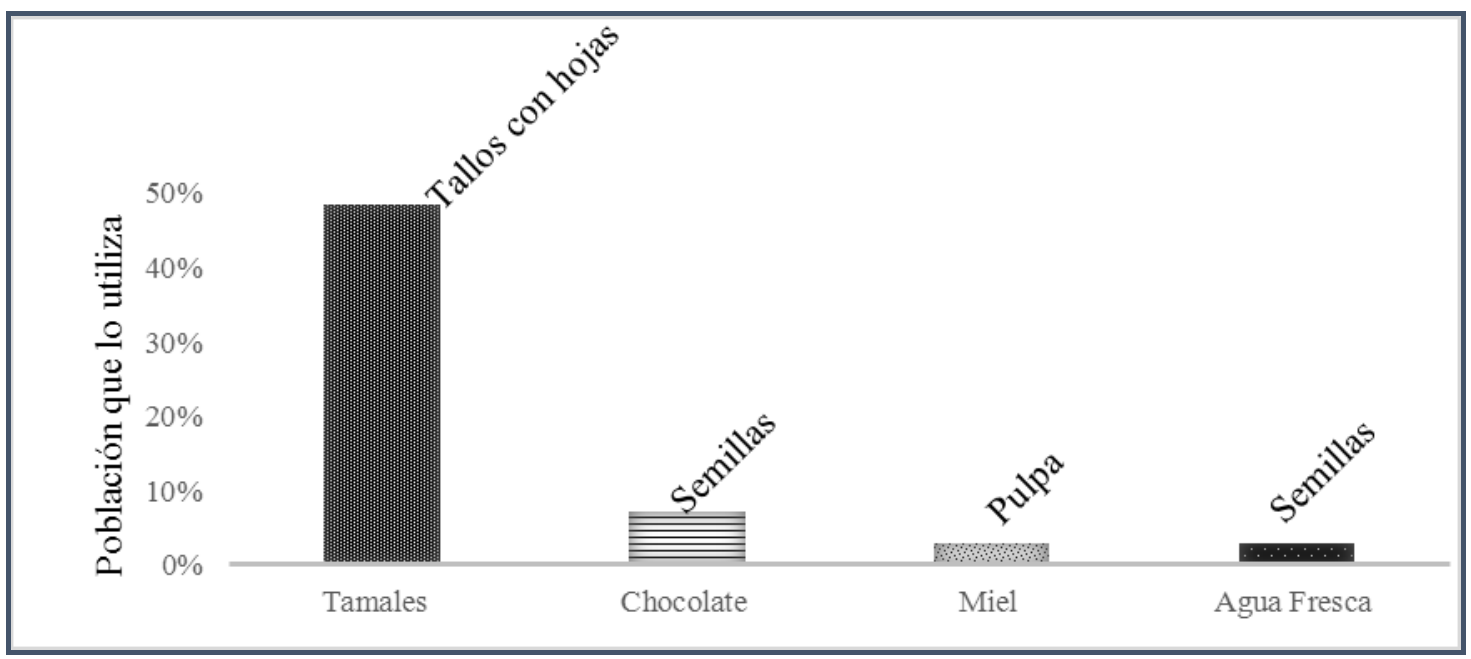

Grafica 3. Uso gastronómico regional de los componentes morfológicos del árbol C. alata.

\section{Uso medicinal del árbol Crescentia alata Kunth}

A partir del conocimiento tradicional las mujeres utilizan todos los componentes del árbol como medicinales (cuadro 3), y sobresale el fruto (100\%), seguido de la flor (15.7\%), la corteza $(5.7 \%)$ y la hoja (1.4\%). La elaboración de productos medicinales con los componentes del árbol podría ser de gran importancia para mejorar los ingresos de las familias en el medio rural por la venta de frutos, hojas y flores. 
Cuadro 3. Uso medicinal del árbol Crescentia alata Kunth.

\begin{tabular}{|l|l|l|c|}
\hline $\begin{array}{c}\text { Componente } \\
\text { morfológico del árbol }\end{array}$ & \multicolumn{1}{|c|}{$\begin{array}{c}\text { Enfermedad } \\
\text { que controla }\end{array}$} & \multicolumn{1}{|c|}{ Como se prepara } & $\begin{array}{c}\text { Días de } \\
\text { tratamiento }\end{array}$ \\
\hline \multirow{5}{*}{ Fruto (pulpa) (100\%) } & Tos (52.9\%) & -hervir con azúcar o piloncillo* & $5-8$ \\
\cline { 2 - 4 } & Golpes (51.4\%) & $\begin{array}{l}\text {-hervir y aplicar en el sitio del golpe } \\
\text {-hervir con azúcar o piloncillo }\end{array}$ & $5-10$ \\
\cline { 2 - 4 } & Gripa (21.4\%) & -hervir con azúcar o piloncillo & $5-7$ \\
\cline { 2 - 4 } & Inflamación (15.7\%) & -hervir con azúcar o piloncillo & $7-15$ \\
\cline { 2 - 4 } & Tumores (12.9\%) & $\begin{array}{l}\text {-tostar la semilla y molerla para tomar } \\
\text { como chocolate } \\
\text {-hervir con azúcar o piloncillo }\end{array}$ & $7-30$ \\
\cline { 2 - 4 } & Dolor (7.1\%) & -hervir con azúcar o piloncillo & $2-8$ \\
\cline { 2 - 4 } & Agruras (1.4\%) & -hervir con azúcar o piloncillo & 6 \\
\cline { 2 - 4 } & Granos (1.4\%) & -hervir con azúcar o piloncillo & $2-9$ \\
\hline & Tos (17.1\%) & -hervir con piloncillo & $3-8$ \\
\cline { 2 - 4 } & Dolores (4.3\%) & $\begin{array}{l}\text {-hervir con piloncillo } \\
\text {-precocer y macerar para extraer el jugo } \\
\text { y aplicar en el oído. }\end{array}$ & $5-8$ \\
\hline & Gripa (2.9\%) & -hervir con piloncillo & $5-7$ \\
\cline { 2 - 4 } & Golpes (1.4\%) & -hervir con piloncillo & $5-7$ \\
\hline \multirow{5}{*}{ Corteza (5.7\%) } & Tos (4.3\%) & -hervir y endulzar con miel de abeja & 7 \\
\cline { 2 - 4 } & Inflamación (1.4\%) & $\begin{array}{l}\text {-hervir y endulzar con miel de abeja o } \\
\text { azúcar }\end{array}$ & 7 \\
\cline { 2 - 4 } & Bronquitis (1.4\%) & $\begin{array}{l}\text {-hervir y endulzar con miel de abeja o } \\
\text { azúcar }\end{array}$ & -hervir y endulzar con miel de abeja \\
\hline Hoja (1.4\%) & Tos (1.4\%) & & \\
\hline
\end{tabular}

*Piloncillo o panela: Preparado del jugo de la caña de azúcar, hervido, moldeado y puesto a secar hasta que se solidifica (\%): Uso de cada componente del árbol y nivel de uso para cada enfermedad

\section{Número de árboles y medidas dasométricas de Crescentia alata Kunth}

El número de árboles dispersos en la pradera y en la cerca viva fue de 218 y 224, respectivamente (cuadro 4). Las medidas dasométricas (DAP y Altura) observadas refieren que los productores seleccionan solamente árboles adultos y dejan una baja proporción de árboles jóvenes producto de la regeneración natural $(<10 \mathrm{~cm}$ DAP), esto puede estar relacionado con las prácticas de manejo como el uso de herbicidas y chapeos.

Cuadro 4. Número de árboles y medidas dasométricas de Crescentia alata identificados en las praderas y en las cercas vivas.

\begin{tabular}{lcccc}
\hline Distribución & $\begin{array}{c}\text { Número árboles } \\
>\mathbf{1 0} \text { cm DAP }\end{array}$ & $\begin{array}{c}\text { Árboles jóvenes } \\
<\mathbf{1 0} \text { cm de DAP }\end{array}$ & $\begin{array}{c}\text { DAP } \\
(\mathbf{c m})\end{array}$ & $\begin{array}{c}\text { Altura } \\
(\mathbf{m})\end{array}$ \\
\hline Dispersos en praderas & $218^{*}$ & 68 & $46.8 \pm 25.3$ & $13.0 \pm 6.3$ \\
Cercas vivas & $224^{* *}$ & 10 & $42.3 \pm 12.1$ & $12.3 \pm 3.5$ \\
\hline
\end{tabular}

*55 transectos de 1 ha, **55 transectos de 100 m lineales, + árboles jóvenes como regeneración natural, \pm desviación estándar, DAP: diámetro del árbol a $130 \mathrm{~cm}$ de altura de la base del suelo. 


\section{Características de los frutos}

En el cuadro 5 se presenta la caracterización del peso y del diámetro de los frutos de $C$. alata (n $=300$ observaciones), también se muestra el peso promedio de las estructuras: cáscara, pulpa más semillas, sólo pulpa y sólo semillas. El numero promedio de semillas por fruto fue de 538.6 \pm 238.5 y estuvo determinado positivamente $(\mathrm{P}<0.0001)$ por el peso $(\mathrm{r}=0.667)$ y el diámetro $(\mathrm{r}=0.657)$ del fruto.

Cuadro 5. Medidas de diámetro y pesos en base seca y su correlación con el número de semillas de los frutos de Crescentia alata Kunth

\begin{tabular}{|c|c|c|c|c|c|c|c|}
\hline \multirow{2}{*}{ Variable } & \multirow{2}{*}{$\begin{array}{l}\text { Diámetro } \\
(\mathbf{c m})\end{array}$} & \multicolumn{5}{|c|}{$\begin{array}{l}\text { Peso (g en base seca) del fruto completo y sus fracciones } \\
\text { (cáscara, pulpa y semillas) }\end{array}$} & \multirow{2}{*}{$\begin{array}{c}\text { Semilla } \\
\left(n^{\circ}\right)\end{array}$} \\
\hline & & Fruto & Cáscara & Pulpa con semilla & Pulpa & Semilla & \\
\hline Media & 9.3 & 294.5 & 109.2 & 204.3 & 128.1 & 57.2 & 538.6 \\
\hline $\mathrm{DE}$ & 1.3 & 125.7 & 58.6 & 105.9 & 74.6 & 35.9 & 238.5 \\
\hline CV (\%) & 14 & 43 & 54 & 52 & 58 & 63 & 44 \\
\hline
\end{tabular}

$\mathrm{DE}=$ desviación estándar, $\mathrm{CV}=$ coeficiente de variación $(\%)$.

\section{DISCUSIÓN}

Los productores tienen un amplio conocimiento sobre el árbol de $C$. alata, coincidiendo con los datos reportados por Solares (2004) sobre la distribución del árbol en todo el estado de Morelos. En otros estudios realizados por Olivares et al., (2016) en el sur del estado de México reportan que $C$. alata fue el árbol más abundante $(59.1 \%)$ y frecuente $(83.3 \%)$ en áreas destinadas a actividades agropecuarias con nueve usos tradicionales. Si además consideramos los usos artesanales de $C$. alata y su importancia en la medicina tradicional y la alimentación de las familias ubican a esta especie arbórea como una de las más importantes en la selva baja caducifolia predominante en la región.

\section{Meses del año en que hay producción de flores y fruto}

Crescentia alata es una especie que produce frutos la mayor parte del año, esto facilita su regeneración natural por su abundante producción de semilla y su dispersión por el ganado después de consumirlo (Villanueva et al., 2003; Bass, 2004), siendo esta una ventaja para preservar la especie en las zonas de pastoreo. En Nicaragua la producción de frutos se distribuye a lo largo de todo el año (Cordero y Boshier, 2003), aunque la cosecha se concentra en dos periodos: primero (agosto-octubre) y segundo (diciembre-abril), con una producción promedio de 60 a 80 frutos por árbol, los cuales se encuentran en diversos grados de desarrollo (CATIE, 2003; Niembro et al., 2010; Rodríguez, 2011).

\section{Beneficios del árbol}

Aporta beneficios múltiples y es un componente importante de la vegetación natural en la zona, con buena adaptación a las condiciones ambientales locales. Estos resultados coinciden con los de Olivares et al. (2016) que reportaron nueve usos complementarios, sobresaliendo sombra, medicinal, árboles en la cerca viva y consumo humano. La preferencia de los productores por ciertas especies depende de los servicios y productos que el árbol brinda a la UP y se convierten de interés para implementar y desarrollar prácticas silvopastoriles (Dagang y Nair, 2003). Crescentia alata conserva la biodiversidad en los sistemas de producción al ampliar hábitats para los animales silvestres, al tiempo que los árboles de esta especie funcionan como corredores biológicos en las cercas vivas (Harvey y Haber, 1999, Villanueva et al., 2003). Los 
conocimientos proporcionados por productores y amas de casa permiten identificar a C. alata como un árbol de usos múltiples tanto en la actividad ganadera como para la obtención de utensilios y fuente de alimento para el hombre.

\section{Alimento para los animales}

A pesar de que el $37.1 \%$ de los productores reportan que los animales consumen el follaje y los frutos en las áreas de pastoreo, es nulo el uso de ambos para alimentar a los animales en un sistema de corte y acarreo. Así mismo, ningún ganadero utiliza al follaje o frutos para elaborar suplementos. Estos resultados son diferentes de los reportados por Zamora et al. (2001) y Bass (2004), quienes encontraron que los productores recolectan o compran frutos de C. alata para alimentar al ganado en la época seca. Aunque son pocos los estudios a la fecha se ha confirmado el potencial forrajero de $C$. alata, sobre la composición nutricional del follaje y los frutos (Rojas et al., 2014) y la respuesta productiva en corderos (Rojas et al. 2012; Rojas et al., 2013; Rojas et al., 2015) y bovinos (Botero y De la Ossa, 2011).

\section{Uso maderable}

La madera es considerada dura por el $74.3 \%$ de los productores, coincidiendo con los resultados de Solares (2004), en donde el 55\% de la trocería de C. alata se clasificó en la categoría "A", que corresponde a primera calidad. Al ser su dureza de mediana a alta tiene la característica necesaria para fabricar artículos donde se requiere mucho esfuerzo y fricción, presenta buena elasticidad para elaborar mangos para herramientas de tipo corto y largo, y permite un acabado fino en la elaboración de muebles. Sin embargo, como poste muerto en las cercas no ha tenido buena aceptabilidad por no resistir el contacto con la humedad del suelo.

\section{Artesanal y ornamental}

Existen reportes que refieren que el fruto es empleado para elaborar jícaras que en muchas ocasiones son talladas y decoradas con pinturas (CATIE, 2003; Rojas, 2006; Fernández, 2009). De igual manera, Solares (2004) reporta que los artesanos elaboran maracas seleccionando frutos de tamaño pequeño y forma circular. Sin embargo, para muchos productores y amas de casa no es atractivo su uso ornamental (Rojas et al., 2010), lo cual puede estar asociado al lento crecimiento de la especie y a que únicamente el $8.6 \%$ de los productores siembran árboles de $C$. alata (cuadro 2).

\section{Alimentación humanos}

En el estado de Guerrero se prepara una gran variedad de comidas y bebidas a base de las hojas y frutos de árboles nativos, que tienen gran aceptación en la población. Estos resultados coinciden con los de Figueroa y Bressani (2000), quienes reportan que en Guatemala se prepara agua fresca a partir de las semillas de $C$. alata (tipo horchata). De igual manera, en la región de Tierra Caliente de Michoacán los tamales nejos elaborados con masa de maíz moldeada con hojas de plátano, son cocidos en un medio acuoso adicionado con hojas y tallos tiernos de Crescentia alata, producto que es servido con mole rojo o verde platillo tradicional de la región (Fernández, 2009; Peralta, 2012). También la semilla extraída de los frutos fue utilizada para la elaboración de polvo utilizado como chocolate en la preparación de infusiones para tomar, así como en la preparación de agua fresca con características similares a el agua de horchata para el consumo de la población (Fernández, 2009; Peralta, 2012).

\section{Uso medicinal}

Crescentia alata es importante en la medicina tradicional (Martínez et al., 2012), ya que se utiliza para tratar enfermedades respiratorias (bronquitis, asma, tos, gripa y tuberculosis), golpes, dolores (actividad antiinflamatoria), tumores (cáncer), problemas digestivos (disentería), erupciones cutáneas (granos) y estimulante del sistema inmunológico, siendo el fruto el componente más utilizado para este fin. Estos resultados coinciden con estudios realizados en México (Rojas et al., 2001; Solares, 2004; Waizel y Waizel, 2009; Magaña et al., 2010; Alonso et al., 2016) y en otros países de América, en donde el árbol es nativo (Beyra et al., 2004; Fernández, 2009; Gómez et al., 2011). La actividad antiinflamatoria de esta especie 
está relacionada a su contenido de flavonoides kaempferol y kaempferol 3- $O$-rutinoside (Autore et al., 2001; Valladares y Ríos, 2006). De igual forma se ha evaluado la actividad antibacteriana del fruto (Espitia et al., 2011). Pío et al. (2013) reportan que los extractos hexánicos y clorofórmicos fueron más activos contra cepas de Streptococcus y S. aureus y contra bacterias Gram negativas los extractos hexánicos registraron actividad contra E. coli, Salmonella y Shigella spp. En tanto que el extracto Clorofórmico fue más activo contra Salmonella thyphi y Shigella spp. Estudios realizados en la especie Crescentia identificaron alcaloides, taninos y flavonoides como componentes antimicrobianos (Rojas et al., 2001). Cada día se presta más atención al estudio de las plantas medicinales, tanto en la práctica de la medicina complementaria como en el ámbito académico. La Organización Mundial de la Salud reporta que el $80 \%$ de la población mundial utiliza las plantas como principal recurso medicinal (Beyra et al., 2004). El uso en la medicina tradicional de esta especie puede ser relevante en las comunidades del medio rural, ya que en el futuro pueden mejorar sus ingresos económicos al comercializar los frutos y otros componentes morfológicos de C. alata.

\section{Número de árboles y medidas dasométricas}

El número de árboles dispersos en las praderas y en arreglo de cercos vivos (218 y 224) se puede considerar bajo (cuadro 4), siendo inferiores los resultados de Olivares et al. (2016) quienes encontraron 172 árboles dispersos en la pradera y 12.9 en las cercas vivas. Este bajo número de árboles puede ser debido a la aplicación de herbicidas y a los chapeos que realizan los ganaderos como prácticas de control de malezas (Camargo et al., 2000). Crescentia alata muestra características que facilitan su regeneración natural, como la abundante producción de semilla y la capacidad de dispersión por el ganado después del consumo, pero tiene como desventaja su lento crecimiento (Villanueva et al., 2003).

\section{Peso y correlación de los frutos}

Se puede apreciar (cuadro 5) que el árbol produce frutos de diferentes tamaños (Janzen, 1982), lo cual es independiente de la fase de maduración, corroborando que a mayor peso y diámetro del fruto se obtienen mayor contenido de pulpa y de semillas totales, sin tomar en cuenta la viabilidad de estas (Janzen, 1982; Flórez, 2012). Aguirre et al. (2012) concluyen que, en el estado de Yucatán, la población selecciona los árboles de Crescentia cujete con base en la forma y tamaño de los frutos. Por lo anterior podemos inferir que los productores pueden seleccionar los árboles de $C$. alata de acuerdo a sus necesidades, por ejemplo, árboles que provean frutos de tamaño pequeño para elaborar artesanías como maracas o arboles con frutos de mayor tamaño para la elaboración de jícaras, obtener mayor cantidad de pulpa para uso medicinal, mayor número de semillas que se puedan comercializar para elaborar sustituto de chocolate y la elaboración de agua fresca para el consumo de la población.

\section{CONCLUSIONES}

El árbol de cirián ( $C$. alata) es conocido por todos los productores. La resistencia a la quema y al pisoteo del ganado, así como la producción de frutos en dos temporadas del año con abundante semilla ha permitido la persistencia del árbol en áreas agrícolas con ejemplares dispersos en las praderas y en arreglo de cercos vivos. El uso de los frutos como alimento para el ganado ha permitido la dispersión de la semilla en el campo, factor importante para mantener la densidad del árbol en los potreros. El árbol Crescentia alata es importante en la economía de la población por que la madera es preferida para la elaboración de sillas, monturas y el fruto para la elaboración de jícaras y el tratamiento de algunas enfermedades (tos), abatir el dolor y la inflamación por golpes; las hojas con tallos tiernos en la preparación de tamales nejos y las semillas son utilizadas para elaborar sustituto de chocolate para preparar infusiones para tomar en forma de té y/o como agua fresca. 


\section{LITERATURA CITADA}

Aguirre, D.X., L.E. Eguiarte, A. González and A. Casas, 2012. "Round and large: morphological and genetic consequences of artificial selection on the gourd tree Crescentia cujete by the Maya of the Yucatan Peninsula, Mexico". Ann. Bot., 109: 1297-1306.

Alonso, C.A.J., M.C. Juárez y N. Campos, 2016. "Medicinal Plants from Mexico, Central America, and the Caribbean Used as Immunostimulants". Evid-Based Complementary Altern. Med., 1-15 p. http://dx.doi.org/10.1155/2016/4017676

Autore, G., L. Rastrelli, M.R. Lauro, S. Marzocco, R. Sorrentino, U. Sorrentino, A. Pinto and R. Aquino, 2001. "Inhibition of nitric oxide synthase expression by a methanolic extract of Crescentia alata and its derived flavonols". Life Sci., 70: 523-534.

Bass, J., 2004. "Incidental Agroforestry in Honduras: The jícaro tree (Crescentia spp.) and pasture land use". J. Lat. Am. Geogr., 3: 67-80.

Beyra, A., M.C. León, E. Iglesias, D. Ferrándiz, R. Herrera, G. Volpato, D. Godínez, M. Guimarais, y R. Álvarez, 2004. "Estudios etnobotánicos sobre plantas medicinales en la provincia de Camagüey (Cuba)". Anales Jard. Bot. Madrid, 61: 185-204.

Botero, L.M. y J. De La Ossa, 2011. "Consumo suplementario de ensilaje salino de frutos maduros de Totumo (Crescentia cujete) en ganado vacuno de doble propósito". Rev. Zoot. Trop., 29: 293-300.

Camacho, M., 2000. "Parcelas permanentes de muestreo en bosque natural tropical". Turrialba, CR, CATIE. 52 p.

Camargo, J.C., M. Ibrahim, E. Somarriba, B. Finegan, y D. Current, 2000. "Factores ecológicos y socioeconómicos que influyen en la regeneración natural de laurel en sistemas silvopastoriles del trópico húmedo y subhúmedo de Costa Rica". Agrofor. Am., 7: 46-52.

CATIE, 2003. "Árboles de Centroamérica: un manual para extensionistas. Crescentia alata”. Editores: Jesús Cordero, David Boshier. Contribuidores: A. Barrance, Centro Agronómico Tropical de Investigación y Enseñanza, Oxford Forestry Institute, Great Britain. Forestry Research Programme. Editor: Bib. Orton IICA / CATIE. Pp 497-502.

Cordero, J. y D. Boshier, 2003. "Árboles de Centroamérica: un manual para extensionistas". Centro Agronómico de Investigación y Enseñanza - CATIE, Oxford, Reino Unido. $1079 \mathrm{pp}$.

Couttolenc, B.E., J.A. Cruz, E. Cedillo y A. Musálem, 2005. “Uso local y potencial de las especies arbóreas en Camarón de Tejeda, Veracruz". Rev. Chapingo Ser. Cie., 11: 45-50.

Dagang, P.K.D. and K.R. Nair, 2003. "Silvopastoral research and adoption in Central America: recent findings and recommendations for future directions". Agrofor. Syst., 59: 149-155.

Espitia, B.J.E., H. de R. Duran, J. Fandiño, F. Díaz y H.A. Gómez, 2011. "Química y biología del extracto etanólico del epicarpio de Crescentia cujete L. (totumo)”. Rev. Cubana Plant. Med., 16: 337-346.

Fernández, C.C., 2009. "Plantas comestibles de Centroamérica". 1 $1^{\mathrm{a}}$ ed. Santo Domingo de Heredia, Costa Rica: Instituto Nacional de Biodiversidad, INBio. Pp. 117-119.

Figueroa, M.S.A. y R. Bressani, 2000. "Recursos alimenticios vegetales con potencial de explotación agroindustrial de Guatemala. Elaboración de leche vegetal a partir de la semilla del fruto de morro (Crescentia alata)". Arch. Latinoam. Nutr., 50: 164-170.

Flórez, J.E., 2012. "Evaluación de pulpa de totumo (Crescentia cujete L) ensilada en dos estados de maduración como alternativa en alimentación bovina". Temas Agrarios, 17: 44-51.

Fragoso, L.C., 1990. "Monografía del Estado de Guerrero, sur amate de mar y montaña”. SEP, México, D.F. 237p.

Gómez, E.H., F. Díaz, L. Franco, J. Mercado, J. Guzmán, D. Medina and R. Gaitán, 2011. "Folk medicine in the northern coast of Colombia: an overview". J. Ethnobiol. Ethnomed., 7: 27.

Harvey, C.A. and A. Haber, 1999. "Remnant trees and the conservation of biodiversity in Costa Rica pastures”. Agrofor. Syst., 44: 37-68. 
Hernández, H.J.E., F.J. Franco y R.M. Pedraza, 2002. "Principales frutos en el trópico y sub trópico generadores de esquilmos con potencial para la alimentación de rumiantes en México". Rev. Prod. Anim., 14: 71-73.

Janzen, D.H., 1982. "Fruit traits, and seed consumption by rodents, of Crescentia alata (bignoniaceae) in Santa Rosa National Park, Costa Rica”. Amer. J. Bot., 69: 12581268.

Magaña, A.M.A., L.M. Gama y R. Mariaca, 2010. "El uso de las plantas medicinales en las comunidades Maya-Chontales de Nacajuca, Tabasco, México”. Polibotánica, 29: 213-262.

Martínez, P.A., A. López, G. Muñoz y J.A. Cuevas, 2012. "Plantas silvestres útiles y prioritarias identificadas en la mixteca poblana, México". Acta Bot. Mex., 98: 73-98.

Niembro, R.A., M. Vázquez and O. Sánchez, 2010. “Árboles de Veracruz 100 especies para la reforestación estratégica. Comisión del Estado de Veracruz de Ignacio de la Llave". Centro de Investigaciones Tropicales. Secretaría de Educación del Estado de Veracruz.

Olivares, J., S. Rojas, F. Avilés, L.M. Camacho, M. Cipriano, R. Jiménez and F. Quiroz, 2016. "Uses of non-leguminous trees in silvopastoral systems in the south of the State of Mexico". Ecosist. Rec. Agropec., 3:193-202.

Palma, J.M., 2006. "Los sistemas silvopastoriles en el trópico seco mexicano". Arch. Latinoam. Prod. Anim., 14: 95-104.

Peralta, V.G., 2012. "Contribución al conocimiento del árbol de Cirián (Crescentia alata Kunth) variabilidad, selección, morfología, citogenética y fitoquímica”. Tesis de Doctorado. Colegio de Postgraduados. Montecillos Estado de México. 198 p.

Pinto R., H. Gómez, B. Martínez, A. Hernández, F.J. Medina, R. Gutiérrez, E. Escobar y J. Vázquez, 2005. “Árboles y arbustos forrajeros del sur de México". Pastos y Forrajes, 28: 87-98.

Pío, L.J.F., P. Díaz, A. López, M. de J. Uribe, K. Willms, G. López, J. Montes y F. Delgado, 2013. "Actividad antibacteriana de extractos de frutos de nanchi (Byrsonima crassifolia (L.) Kunth), arrayán (Psidium sartorianum (O. Berg) Nied.) y ayale (Crescentia alata Kunth)". Bol. Latinoam. Caribe Plant. Med. Aromat., 12: 356-364.

Rodríguez, B.M., 2011. "Efecto de los árboles aislados sobre las características del suelo en sistemas silvopastoriles en Rivas, Nicaragua”. Tesis Magister Scientiae en agroforestería Tropical. Centro de agronomía tropical de investigación y enseñanza. 77 pp.

Rojas, S.R., 1987. “Guía para realizar investigaciones sociales”. Ed. Plaza y Valdés.

Rojas, H.S., F. Avilés, O.A. Castelán, A. García y J. Olivares, 2010. "Tipificación de los sistemas de producción de ganado e importancia de los árboles no leguminosos en la zona rural del sur del Estado de México”. In: Marcof, A.C.F. Los grandes retos para la ganadería: Hambre, Pobreza y Crisis Ambiental. Chapingo, Estado de México. Pp 115124.

Rojas, G., J. Lévaro, J. Tortoriello and V. Navarro, 2001. "Antimicrobial evaluation of certain plants used in Mexican traditional medicine for the treatment of respiratory diseases". J. Ethnopharmacol., 74: 97-101.

Rojas, H.S., F. Aviles, O.A. Castelan, A. Garcia, J. Olivares and M.T. Valencia, 2012. "Chemical Composition, In vitro Digestibility of Foliage Guazuma ulmifolia and Crescentia alata and its Use in Feeding Lambs". Pak. J. of Nutr., 11:1139-1145.

Rojas, H.S., J. Olivares, I. Gutiérrez, R. Jiménez, F. León y A. Córdova, 2013. "Use of Crescentia alata and Guazuma ulmifolia fruits in lamb feeding in subtropical region of Guerrero, Mexico". Rev. Científ. FCV-LUZ, 23: 157-162.

Rojas, H.S., J. Olivares, V. López, E. Hernández, M. T. Valencia, I. Gutiérrez y F. Quiroz, 2014. "Composición nutricional y aceptabilidad de ensilado de frutos de Cirián (Crescentia alata) en caprinos". XLI Reunión de la Asociación Mexicana para la Producción Animal y Seguridad Alimentaria A.C. (AMPA). Mérida, Yucatán, México del 2 al 4 de Julio. Pp. 253-257.

Rojas, H.S., J. Olivares, F. Aviles, A. Villa, A. Reynoso, and L.M. Camacho, 2015. "Productive response of lambs fed Crescentia alata and Guazuma ulmifolia fruits in a tropical region of Mexico". Trop. Anim. Health Prod., 47: 1431-1436. 


\section{Recibido:}

11/marzo/2016

Aceptado:

26/septiembre/2016

Rojas, R.F., 2006. “Árboles: mucho más que madera”. Kurú: Revista Forestal (Costa Rica) 3: 1-13.

Statistical Analysis System (SAS). 2001. SAS/STAT. In: Guide for Personal Computers Version Ver 9.0. Institute Inc. Cary, NC, USA. pp: 956.

Solares, A.F., 2004. "Etnobotánica y usos potenciales del Cirián (Crescentia alata, H. B. K.) en el estado de Morelos". Polibotánica, 18: 13-31.

Steel, R. y J. Torrie, 1988. "Bioestadística: principios y procedimientos". 622p. $2^{\mathrm{a}}$ ed. McGrawHill, México.

Valladares, M.G. and M.Y. Ríos, 2007. "Iridoids from Crescentia alata". J. Nat. Prod., 70: 100-102

Villacis, J., A. Harvey, I. Muhammad y C. Villanueva, 2003. "Relaciones entre la cobertura arbórea y el nivel de intensificación de las fincas ganaderas en Río Frío, Costa Rica". Agrofor. Am., 10: 17-23.

Villanueva, C., I.C. Harvey y H. Esquivel, 2003. "Tipologías de fincas con ganadería bovina y cobertura arbórea en pasturas en el trópico seco de Costa Rica". Agrofor. Am., 10: 9-16.

Waizel, H.S. y J. Waizel, 2009. "Algunas plantas utilizadas en México para el tratamiento del asma”. An. Orl. Mex., 54:145-71.

Zamora, S., J. García, G. Bonilla, H. Aguilar, A.C. Harvey y I. Muhammad, 2001. "Uso de frutos y follaje arbóreo en la alimentación de vacunos en la época seca en Boaco, Nicaragua". Agrofor. Am., 8: 31-38. 